\title{
TUMOR GLÔMICO NO HÁLUX COM VERRUGA VULGAR ASSOCIADA
}

\author{
Taynara de Mattos Barreto', Robertha Carvalho Nakamura², Talita Batalha Pires dos Santos ${ }^{3}$, Dâmia Leal Vendramini ${ }^{4}$, \\ Thales Pereira de Azevedo 5 , Anna Luiza Bento Dutra ${ }^{5}$ \\ 'Médica Graduada/Medical Graduated, Universidade Federal do Rio de Janeiro (UFRJ) \\ ${ }_{2}^{2}$ Mestre em Dermatologia/Master in Dermatology, Universidade Federal do Rio de Janeiro (UFRJ); Preceptora e \\ Coordenadora/Preceptor and Coordenator, Centro de Estudos da Unha (CEU) do Instituto de Dermatologia Professor \\ Rubem David Azulay da Santa Casa da Misericórdia do Rio de Janeiro (IDPRDA-SCMRJ) \\ ${ }^{3}$ Médica Graduada/Medical Graduated, Centro Universitário de Volta Redonda (UniFOA) \\ ${ }^{4}$ Médica Graduada/Medical Graduated, Universidade do Sul de Santa Catarina (UNISUL) \\ ${ }^{5}$ Médico Graduado/Medical Graduated, Universidade Federal do Rio de Janeiro (UFRJ)
}

RESUMO - O tumor glômico é um tumor mesenquimal incomum originado do glomusneuromioarterial presente na derme reticular, localizado mais frequentemente nas mãos.

Relatamos um caso de mulher, 51 anos, com história de longa duração de alterações no aspecto da unha do hálux esquerdo e dor local intensa. Após diversos tratamentos, já com o diagnóstico de verruga subungueal, foi suspeitada a existência de tumor glômico pela história clínica e testes semiológicos sugestivos. Foi realizada cirurgia, com a confirmação histopatológica das duas hipóteses.

O possível diagnóstico é a soma de história clínica sugestiva, testes ambulatoriais e exames de imagem, contudo somente confirmado pela histopatologia.

O tratamento é cirúrgico, tratando a dor e evitando a recorrência. O presente caso relata associação de diagnósticos (tumor glômico e verruga subungueal) culminando com as importantes alterações no aspecto da unha.

Enfatiza-se neste trabalho a necessidade de avaliar a dor obscura de dígitos para tumor glômico.

PALAVRAS-CHAVE - Doenças do pé; Doenças das unhas; Hálux; Neoplasias da pele; Tumor glômico.

\section{GLOMUS TUMOR WITH ASSOCIATED SUBUNGUAL WART ON HALLUX}

ABSTRACT - Glomus tumor is an uncommon mesenchymal tumor present in the reticular dermis, frequently located in the hands.

We report a case of a woman, 51 years old, with a history of long-lasting changes in the appearance of the left hallux's nail and intense local pain. After several treatments, being already diagnosed the subungual wart, glomus tumor was suspected, because of the suggestive symptoms and clinical tests. Surgery was performed, with histopathological confirmation of both hypotheses.

The possible diagnosis is the sum of suggestive clinical history, clinical tests and imaging tests, but only confirmed by histopathology.

The treatment is surgical, treating pain and preventing recurrence. The present case reports associated diagnoses (glomus tumor and subungual wart) culminating with the major changes in the appearance of the nail.

This paper emphasizes the need to suspect the diagnostic of glomus tumor on cases of obscure pain in fingers or toes.

KEY-WORDS - Hand dermatoses; Fibroma.

Conflitos de interesse: Os autores declaram não possuir conflitos de interesse.

No conflicts of interest.

Suporte financeiro: O presente trabalho não foi suportado por nenhum subsídio ou bolsa. 


\title{
Caso Clínico
}

\begin{abstract}
No sponsorship or scholarship granted.
Direito à privacidade e consentimento escrito / Privacy policy and informed consent: Os autores declaram que pediram consentimento ao doente para usar as imagens no artigo. The authors declare that the patient gave written informed consent for the use of its photos in this article.
\end{abstract}

Recebido/Received - Novembro/November 2013; Aceite/Accepted - Janeiro/January 2014

Correspondêncią

Dr. a Taynara de Mattos Barreto

Rua Capitão Menezes, 330 apto. 701

Praça Seca Rio de Janeiro - RJ Brasil

CEP: 21320-040

Email: taynaramb@gmail.com

\section{INTRODUÇÃO}

O tumor glômico foi inicialmente reportado por Wood em 1912, mas foram Barre e Masson, em 1924, que $\circ$ descreveram um hamartoma vascular benigno originado do glomus neuromioarterial presente na derme reticular ${ }^{1,2}$. Sua localização mais freqüente é a área subungueal nas mãos, sendo frequentemente observado como lesão única na falange distal dos dedos $^{3,4}$. Representa de um até $4,5 \%$ das neoplasias que acometem as mãos ${ }^{3}$. Também pode ocorrer nos dedos dos pés, embora seja menos usual ${ }^{4}$.

O tumor é mais frequente em mulheres, ocorrendo em média entre a terceira e quinta década de vida. Apresenta-se clinicamente com dor pulsátil intensa que pode ser espontânea ou desencadeada por pressão ou trauma e alterações de temperatura ${ }^{2,3}$. Pode apresentar nódulo ou pápula de coloração azul-avermelhada de até cerca de $2 \mathrm{~cm}$ ou pode haver presença de eritroníqua longitudinal com ou sem distrofia ungueal. Quando ocorre distrofia ungueal, geralmente apresenta-se com onicólise, fenda ungueal longitudinal ou fragmentação da lâmina ungueal, mas outras alterações também podem ocorrer. A tríade dor paroxística, sensibilidade local e hipersensibilidade a mudanças de temperatura deve levar a pensar na hipótese de tumor glômico ${ }^{5}$.

O diagnóstico pode ser feito pelo exame clínico com seus achados físicos, pela história da doença, pela semiologia e pelos exames complementares. A semiologia engloba testes como o Love, em que a localização do tumor é determinada quando há dor no local em que é aplicada pressão com a cabeça de um alfinete; Hildreth, que consiste no alívio da dor com a aplicação de um torniquete proximalmente ao dedo comprometido; e de sensibilidade ao frio ou teste gelo, havendo dor quando há contato do dígito com água gelada ${ }^{3}$.
A dermatoscopia é um exame complementar que vem aumentando sua acurácia ao longo dos anos, principalmente quando a lesão está na localização subungueal. Os exames radiológicos são úteis para o diagnóstico, sendo a radiografia simples positiva em apenas $40 \%$ dos casos, relacionada a tumores maiores e apresentando características erosivas, com invasão óssea. Pode-se realizar a ultrassonografia ou a ressonância magnética nuclear para auxílio diagnóstico na determinação da localização do tumor, tendo a última maior acurácia. A histopalogia é o exame gold stan$\operatorname{dard}^{3,4,6}$.

O tratamento de escolha é cirúrgico, com a excisão completa do tumor, para alívio da sintomatologia dolorosa e prevenção da recorrência ${ }^{3}$.

\section{CASO CLÍNICO}

Mulher, 51 anos, com história de longa duração de alterações no aspecto da unha do hálux esquerdo e dor local intensa, que a impedia de usar calçados fechados ou que ficassem sobre a unha. Foi submetida a diversos tratamentos, inclusive exérese da unha e cauterização por diagnóstico de verruga subungueal lateral, sem melhora do quadro doloroso e havendo piora do com a persistência da verru$\mathrm{ga}$, onicocriptose e onicólise associada. Ao procurar novo tratamento, foi suspeitado tumor glômico pela história de dor episódica latejante e intensa, que ocorria ou piorava ao contato do dedo com água fria.

Ao exame, apresentava aumento da curvatura transversa da unha em forma de telha, com eritroníquia localizada e mal delimitada. Dor à compressão da placa ungueal e ao contato com água gelada. 


\section{Caso Clínico}

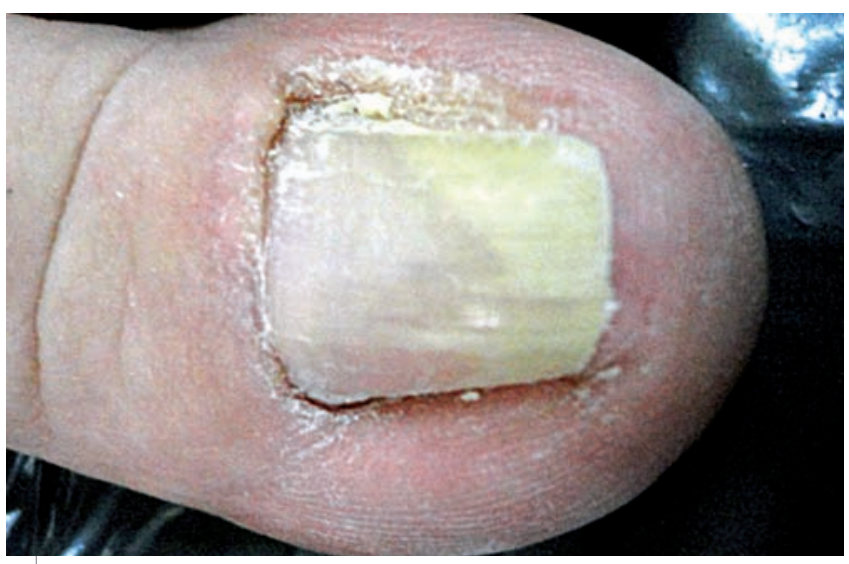

Fig 1 - Unha em telha com mácula eritematosa subungueal medial e hiperceratose lateral.

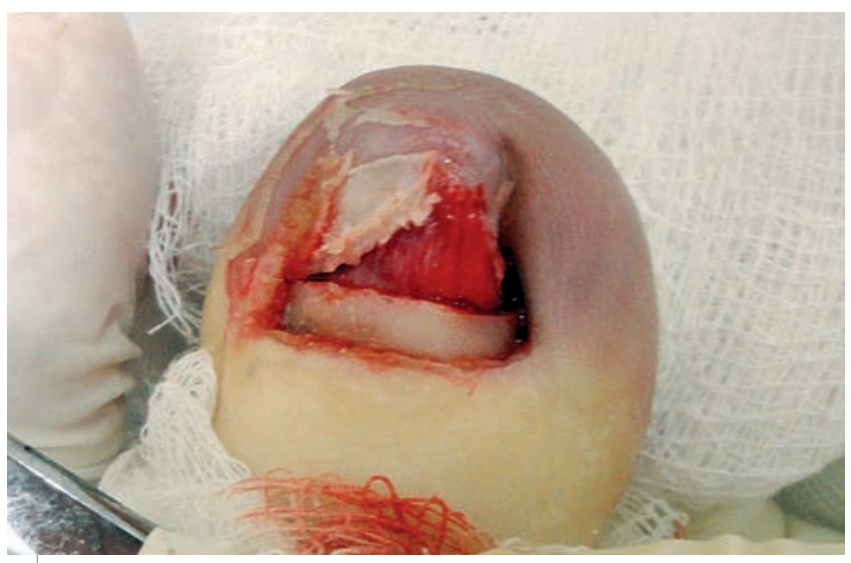

Fig 3 - Durante a cirurgia, após retirada parcial da unha.

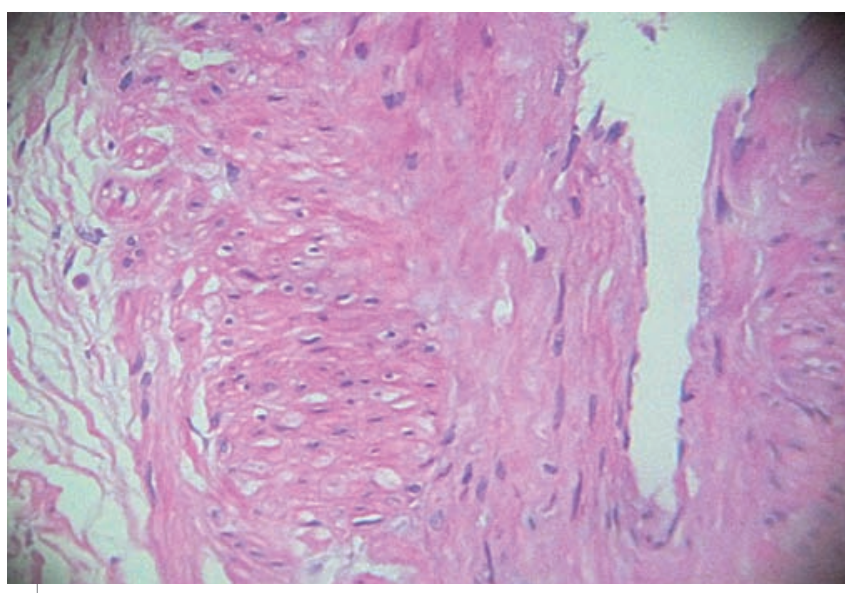

Fig 5 - Derme exibindo espaços delimitados por endotélio e circundados por células glômicas.

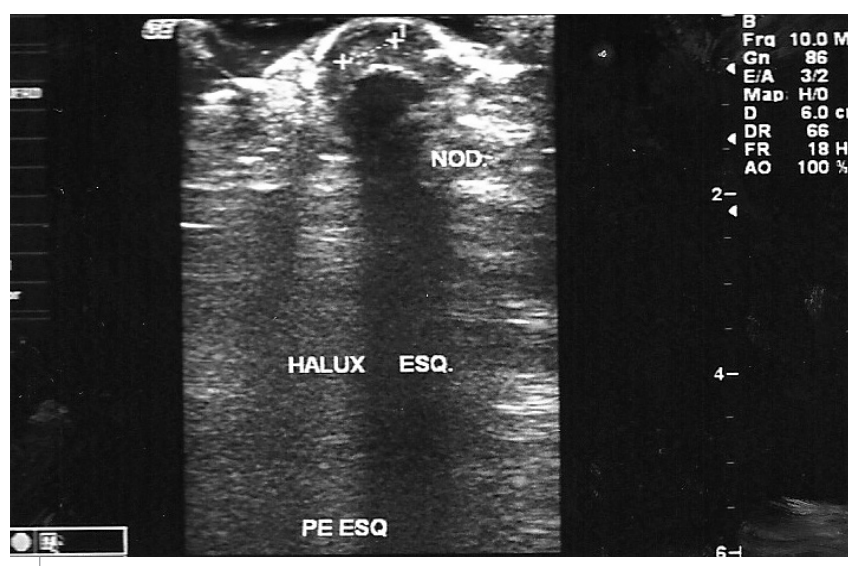

Fig 2 - Imagem de ultrassonografia evidenciando o nódulo subungueal.

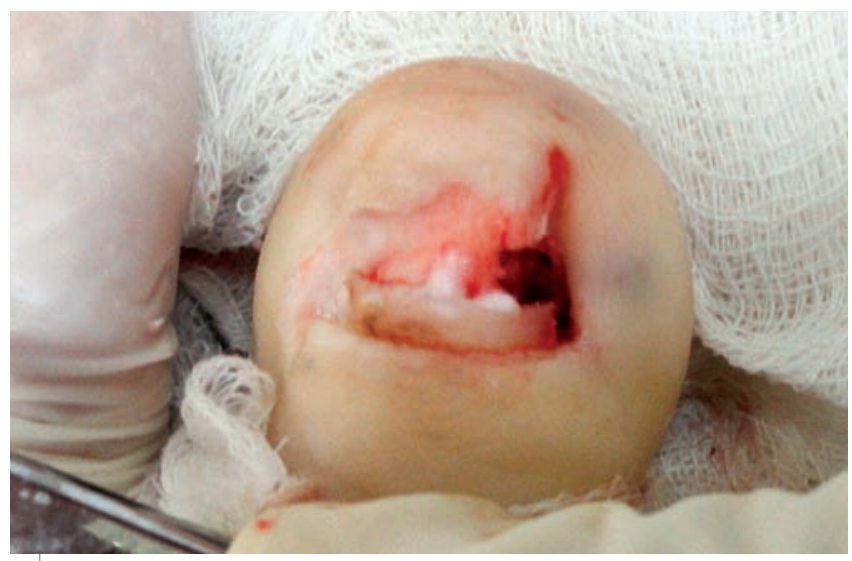

Fig 4 - Ao final da cirurgia, após exérese do tumor glômico e desbridamento da verruga subungueal.

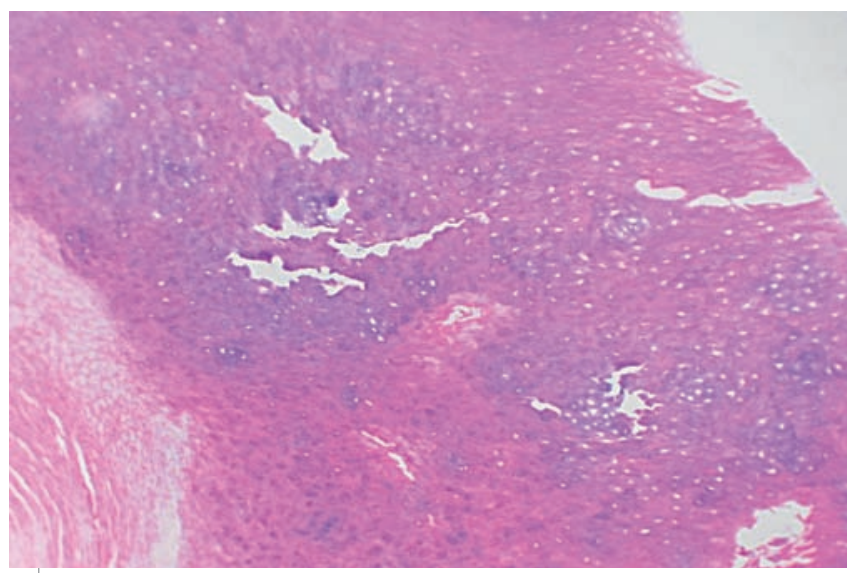

Fig 6 - Observa-se pronunciada hiperplasia digitada do epitélio do leito ungueal, com hiperceratose, hipergranulose e arborização dos cones periféricos. 


\section{Caso Clínico}

Realizou exames complementares, com os seguintes resultados: Radiografia sem alterações ósseas. $\mathrm{Na}$ ultrassonografia, nódulo sólido levemente hipoecoico localizado na região subungueal da falange distal do hálux. Ao Doppler, vasos calibrosos na topografia local. Aspecto compatível com tumor glômico subungueal. A Ressonância Magnética Nuclear não apresentou alterações.

A paciente foi submetida à cirurgia para exérese da lesão, que também abordou a verruga com desbridamento meticuloso, evitando sangramento e a possibilidade de disseminação do vírus. Foi necessária a exérese da parte distal da unha para a abordagem tanto do tumor quanto da parte subungueal da verruga. Ainda, foi abordada a matriz ungueal, para auxiliar na correção do formato da unha. Os materiais das duas lesões foram encaminhados para exame histopatológico com a confirmação do tumor glômico e verruga subungueal.

Após dois meses, a paciente permanece em tratamento com imiquimod para a verruga, mas com melhora completa da sintomatologia álgica relativa ao tumor glômico e completa cicatrização do local.

\section{DISCUSSÃO}

O tumor glômico subungueal é incomum na prática dermatológica, sendo considerado um tumor vascular benigno raro ${ }^{3}$. O local mais comum de encontro desse tipo de tumor não é o hálux e sim, a extremidade distal dos quirodáctilos ${ }^{4}$. $O$ caso relatado é particularmente interessante, pois ele foi diagnosticado e tratado incompletamente por duas vezes, demonstrando que esse tipo de lesão é raramente suspeitada e muitas vezes mal diagnosticada, o que aumenta a morbidade dos pacientes.

A suspeita diagnóstica do tumor glômico deve ser dada por uma combinação de história clínica sugestiva, testes ambulatoriais como o teste de Love e teste de Hildreth positivos, e exames de imagem sugestivos. A partir dessa suspeita há indicação de excisão cirúrgica com posterior análise histopatológica que confirmará a hipótese diagnóstica.

Os exames de imagem são importantes no auxílio diagnóstico do tumor glômico corroborando a hipótese diagnóstica e também auxiliando no planejamento da excisão cirúrgica. $\mathrm{O}$ raio-X anteroposterior e latero-lateral da região é usado em concomitância com a ressonância nuclear magnética (RNM) e/ou ultrassonografia de alta resolução para investigação de comprometimento ósseo com erosão. A RNM proporciona um excelente contraste entre a neoplasia e o tecido normal mas não consegue diferenciar entre tumor glômico e neoplasia sólida em T2, mas, em T1, um sinal baixo acompanhado de um sinal alto é característica de hipervascularização típica do tumor glômico. Já a ultrassonografia possibilita a visualização do local, tamanho e formato do tumor mesmo se este não for palpável. A imagem ultrassonográfica mais característica é de uma área globular hipoecóica de difícil diferenciação para demais tumores hipoecóicos, mas ainda assim é eficaz para o planeamento cirúrgico que é o seu principal objetivo. Vale ressaltar que ela é eficiente para tumores menores do que $2 \mathrm{~mm}$ e em região lateral subungueal ${ }^{6}$.

Tumores subungueais glômicos são mais difíceis de tratar, pois exigem cirurgia meticulosa para preservação da unha. Excisão incompleta é motivo para recorrência da dor. Deformidade da unha pode ser evitada adotando abordagem periungueal ou transungueal, seguida de reparação meticulosa do leito ungueal ${ }^{7,8}$. Posteriormente, para confirmação diagnóstica é necessário realizar uma avaliação anatomopatológica de um fragmento da tumoração e descartar malignidade que pode estar presente em $1 \%$ dos casos $^{3}$. Em nosso caso, uma vez que os exames de imagem (ultrassonografia e ressonância magnética) eram controversos e o tumor era muito pequeno, foi realizada a ressecção em bloco para o histopatologista seriar.

O ponto mais interessante deste caso foi a associação de diagnósticos (tumor glômico e verruga subungueal) culminando com as importantes alterações no aspecto da unha. No início da evolução do caso, a verruga foi o único diagnóstico, não sendo suspeitado o tumor glômico nas primeiras avaliações, sendo a dor atribuída à onicocriptose. A verruga ungueal ou verruga canthi é uma infecção causada pelo papilomavírus humano (HPV), mais comunmente em seus subtipos 1,2 e 4 . Apresenta período de incubação de poucas semanas até muitos meses. Clinicamente apresenta-se como pápula única ou múltipla de superfície ceratósica com pontos enegrecidos localizadas principalmente no hiponíquio e leito ungueal, podendo evoluir para onicodistrofia. Dissemina-se por autoinoculação. $O$ diagnóstico geralmente é clínico, sendo necessária a biópsia em verrugas de longa data para a exclusão de carcinoma de células escamosas. Há diversas opções terapêuticas disponíveis. O tratamento para verruga periungueal é difícil, com dificuldade no processo de cicatrização ${ }^{5}$. 


\section{Caso Clínico}

Ao relatar esse caso, pretendemos enfatizar a necessidade de avaliar a dor obscura de quirodáctilos ou pododáctilos para tumor glômico. Perspicácia clínica, simples testes diagnósticos e se necessários, ultrassonografia e ressonância nuclear magnética devem ser utilizados. Uma vez diagnosticada, excisão completa da lesão e preservação da unha deve ser feita sempre que possível.

\section{REFERÊNCIAS}

1. Tumor Glômico (glomangiomas). In: Azulay RD, editor. Dermatologia. 5a ed.Rio de Janeiro: Guanabara Koogan; 2008.p. 227.

2. Netscher DT, Aburto J, Koepplinger M. Subungual Glomus Tumor. J Hand SurgAm. 2012; 37:821-3.

3. Vanti AA, Cuce LC, Chiacchio ND. Tumor glômico subungueal: estudo epidemiológico e retrospectivo, no período de 1991 a 2003. An Bras Dermatol. 2007; 82(5):425-31.

4. Polo C, Borda D, Poggio D, Asunción J, Peidro L. Glomus tumor of the hallux. Review of the literature and report of two cases. Foot Ankle Surg. 2012; 1889-93.

5. Baran R, Nakamura R. Doenças da Unha: do diagnóstico ao tratamento. $1^{\mathrm{ed}}$. Rio de Janeiro: Guanabara-Koogan; 2011.

6. Chen S, Chen Y, Chen M, Yeow K, Chen H, Wei F. The use of ultrasonography in preoperative localization of digital glomus tumors. Plast Reconstr Surg. 2003; 112(1):115-9.

7. Giele H. Hildreth's test is a reliable clinical sign for the diagnosis of glomus tumours. J Hand Surg. 2002; 27:157-8.

8. Takemura N, Fujii N, Tanaka T. Subungual glomus tumor diagnosis based on imaging. J Dermatol. 2006; 33:389-93. 